\title{
Synthesis of few-layer graphene via microwave plasma-enhanced chemical vapour deposition
}

\author{
Alexander Malesevic ${ }^{1,2,4}$, Roumen Vitchev ${ }^{1}$, Koen Schouteden ${ }^{2}$, \\ Alexander Volodin ${ }^{2}$, Liang Zhang ${ }^{3}$, Gustaaf Van Tendeloo ${ }^{3}$, \\ Annick Vanhulsel ${ }^{1}$ and Chris Van Haesendonck ${ }^{2}$ \\ ${ }^{1}$ VITO Materials, Flemish Institute for Technological Research, Boeretang 200, \\ BE-2400 Mol, Belgium \\ ${ }^{2}$ Laboratory of Solid-State Physics and Magnetism, Katholieke Universiteit Leuven, \\ Celestijnenlaan 200 D, BE-3001 Leuven, Belgium \\ ${ }^{3}$ Electron Microscopy for Materials Science, University of Antwerp, Groenenborgerlaan 171, \\ BE-2020, Antwerp, Belgium \\ E-mail: alexander.malesevic@ @ito.be
}

Received 25 February 2008, in final form 21 May 2008

Published 12 June 2008

Online at stacks.iop.org/Nano/19/305604

\begin{abstract}
If graphene is ever going to live up to the promises of future nanoelectronic devices, an easy and cheap route for mass production is an essential requirement. A way to extend the capabilities of plasma-enhanced chemical vapour deposition to the synthesis of freestanding few-layer graphene is presented. Micrometre-wide flakes consisting of four to six atomic layers of stacked graphene sheets have been synthesized by controlled recombination of carbon radicals in a microwave plasma. A simple and highly reproducible technique is essential, since the resulting flakes can be synthesized without the need for a catalyst on the surface of any substrate that withstands elevated temperatures up to $700^{\circ} \mathrm{C}$. A thorough structural analysis of the flakes is performed with electron microscopy, x-ray diffraction, Raman spectroscopy and scanning tunnelling microscopy. The resulting graphene flakes are aligned vertically to the substrate surface and grow according to a three-step process, as revealed by the combined analysis of electron microscopy and x-ray photoelectron spectroscopy.
\end{abstract}

\section{Introduction}

A single sheet of $\mathrm{sp}^{2}$ hybridized carbon atoms defines the strictly two-dimensional graphene crystal, the elementary building block of graphite. Graphene was first isolated from bulk graphite by ingenious but elaborate exfoliation techniques, which offered the possibility to study its physical properties $[1,2]$. Since then, a large number of publications have reported graphene's exciting electronic properties, including the room temperature anomalous quantum Hall effect [3, 4] and electrons behaving like massless Dirac fermions $[5,6]$. Due to the very high mobility and the possibility to tune the electronic conduction via the field effect, graphene is also a very promising material for future electronic

4 Author to whom any correspondence should be addressed. applications [7-9]. The recent discovery of graphene's ability to detect single gas molecules adsorbed on its surface adds exceptionally sensitive chemical detectors to the list of possible future applications [10].

An obstacle on the road to graphene devices is the lack of a method for mass production. Mechanical exfoliation and epitaxial growth [11] generally produce single, bilayer or trilayer graphene sheets, but are elaborate and not very likely candidates for mass production. Recently, it has been shown that graphite oxide readily exfoliates in water, after which a drop of this suspension can be chemically reduced to yield graphene [12]. This highly reproducible chemical route is among the first techniques to promise mass production of graphene.

Microwave plasma-enhanced chemical vapour deposition (MW PECVD) is a well-known technique for the synthesis of 
carbon nanotubes [13] and carbon nanowalls [14]. Similar foil-like carbon nanostructures were synthesized by RF PECVD [15]. The latter material is typically a synonym for defective nanostructured graphite. We have optimized this technique for the synthesis of high quality, crystalline, freestanding few-layer graphene (FLG), just 4-6 atomic layers thick and up to several micrometres wide. An extensive structural characterization of the as-grown FLG and a study of the growth mechanism is presented.

\section{Experimental methods}

FLG was synthesized in a MW PECVD set-up composed of an Iplas Cyrannus MW plasma source mounted on top of a stainless steel vessel. The plasma source consists of a $6 \mathrm{~kW}, 2.45 \mathrm{GHz}$ cylindrical MW resonator, which couples the MW power through five annular slots in a quartz tube and enables operation either in the TM012 or in the surface wave plasma mode. For all the experiments, the plasma was operated in the TM012 mode. A flexible substrate holder allows free positioning of substrates along the axis of the quartz tube. A broad range of materials was used as substrates, including quartz, silicon, nickel, platinum, germanium, titanium, tungsten, stainless steel, tantalum and molybdenum.

Prior to growth, the substrates are heated for $20 \mathrm{~min}$ in a $2 \mathrm{~kW}$ MW plasma at 40 Torr, using a $\mathrm{H}_{2}$ flow of $200 \mathrm{sccm}$. This plasma is sufficiently powerful to heat the samples from room temperature up to $700{ }^{\circ} \mathrm{C}$, as measured by a thermocouple embedded in the substrate holder. Immediately after the heating step, $\mathrm{CH}_{4}$ mixed with $\mathrm{H}_{2}$ is introduced into the growth chamber with a $\mathrm{CH}_{4} / \mathrm{H}_{2}$ ratio of $1 / 8$ at a total flow rate of $200 \mathrm{sccm}$. Keeping the plasma power and pressure at $2 \mathrm{~kW}$ and 40 Torr, respectively, FLG was grown with this gas mixture for various growth times ranging from 1 to $3000 \mathrm{~s}$.

Scanning electron microscope (SEM) analysis was performed with a JEOL JSM6340F microscope, operated with an acceleration voltage of $5 \mathrm{kV}$. The high-resolution transmission electron microscopy (TEM) micrographs were recorded on a Philips CM30 microscope, operated at $300 \mathrm{kV}$ with a point-to-point resolution around $0.1 \mathrm{~nm}$. Electron diffraction was recorded on a Philips CM20 with an acceleration voltage of $200 \mathrm{kV}$. Scanning tunnelling microscopy (STM) measurements were performed with a commercially available lowtemperature Omicron Nanotechnology STM, operating under ultra-high vacuum conditions at a base pressure in the $10^{-11}$ mbar range. For maximum resolution and stability, all measurements were performed at liquid helium temperature $\left(T_{\text {sample }}=4.5 \mathrm{~K}\right)$. STM topographic imaging was performed in constant current mode by using mechanically cut PtIr (10\% Ir) tips. Tunnelling voltage and current were $0.3 \mathrm{~V}$ and $0.6 \mathrm{nA}$, respectively. Prior to STM investigation, the sample was annealed at moderate temperatures of about $450{ }^{\circ} \mathrm{C}$ to remove surface contamination due to exposure to ambient conditions. For x-ray diffraction (XRD), x-rays were produced with a $\mathrm{Cu} \mathrm{K} \alpha$ target $(\lambda=1.5406 \AA)$ and passed through a monochromator. The XRD patterns were recorded with an accuracy of $0.01^{\circ}$ under appropriate Bragg reflection conditions. Raman measurements were performed with an Ar ion laser with a fixed wavelength of $514 \mathrm{~nm}$, a magnification of $100 \times$ and a liquid-nitrogen-cooled CCD camera detector. The narrow scan X-ray photoelectron spectroscopy (XPS) spectra were recorded with a Thermo Electron Theta Probe, equipped with an $\mathrm{K} \alpha$ monochromator and electron flood gun for charge compensation, using an accumulation of 10 sweeps with pass energy of $100 \mathrm{eV}$.

\section{Results}

SEM micrographs in figures 1(a) and (b) clearly show the typical shape and overall dimensions of the FLG, which is aligned vertically to the substrate surface. This orientation makes the flakes potentially useful in field emission devices. Note that the flakes are so thin that they are semi-transparent for the low energy electron bundle. The average thickness of the flakes varies between 4 and 6 atomic layers, as revealed by TEM analysis in figures 1(c) and (d). TEM clearly reveals that the as-grown FLG are planar structures and not nanocones. The ostensible upward curling at the edges of the individual flakes might be due to internal stress in the few-layer graphene. Electron diffraction in figure 1(e) clearly shows a hexagonal diffraction pattern. Further insight into the crystal structure of an individual flake is provided by STM in figure 1(f), revealing a hexagonal atomic lattice structure.

More details about the crystallinity and quality of the flakes can be obtained from the XRD and Raman spectroscopy data presented in figure 2 . The spectra were recorded from large areas of freestanding as-grown flakes, vertically aligned to the substrate surface. Several distinct peaks, corresponding to known values for graphite, can be identified in the XRD spectrum of FLG. The peak position of the $\mathrm{C}(002)$ and the $\mathrm{C}(110)$ peaks at $26.3^{\circ}$ and $77.7^{\circ}$, respectively, were used to calculate the inter-plane distance between two subsequent graphene sheets and the in-plane interatomic distance between two neighbouring carbon atoms, respectively. Both extracted values of $0.34 \mathrm{~nm}$ for the inter-plane and $0.25 \mathrm{~nm}$ for the in-plane interatomic distance correspond, within experimental error, to the tabulated graphite values. The high degree of crystallinity of the flakes was also confirmed by Raman spectroscopy, which showed a weak D-band around $1350 \mathrm{~cm}^{-1}$, a strong G-band around $1580 \mathrm{~cm}^{-1}$ with a small shoulder, identified as the $\mathrm{D}^{\prime}$-band around $1610 \mathrm{~cm}^{-1}$, and a strong 2D-band around $2700 \mathrm{~cm}^{-1}$. The D-band is disorder-induced and caused by phonon scattering at defect sites and impurities [16], while the $\mathrm{D}^{\prime}$-band originates from finite size effects and from phonon scattering at crystal lattice defects [17]. The G-band is related to phonon vibrations in $\mathrm{sp}^{2}$ carbon materials. For a qualitative analysis of graphitic structures, the intensity ratio of the D-band to the G-band is measured and denoted as the $R$ value, which is by definition inversely proportional to quality and is ideally zero for highly ordered pyrolitic graphite [18]. The $R$ value varies between 0.20 and 0.30 for our FLG, depending on the growth conditions. This measured value can be interpreted as an upper limit, since a substantial contribution to the D-band comes 

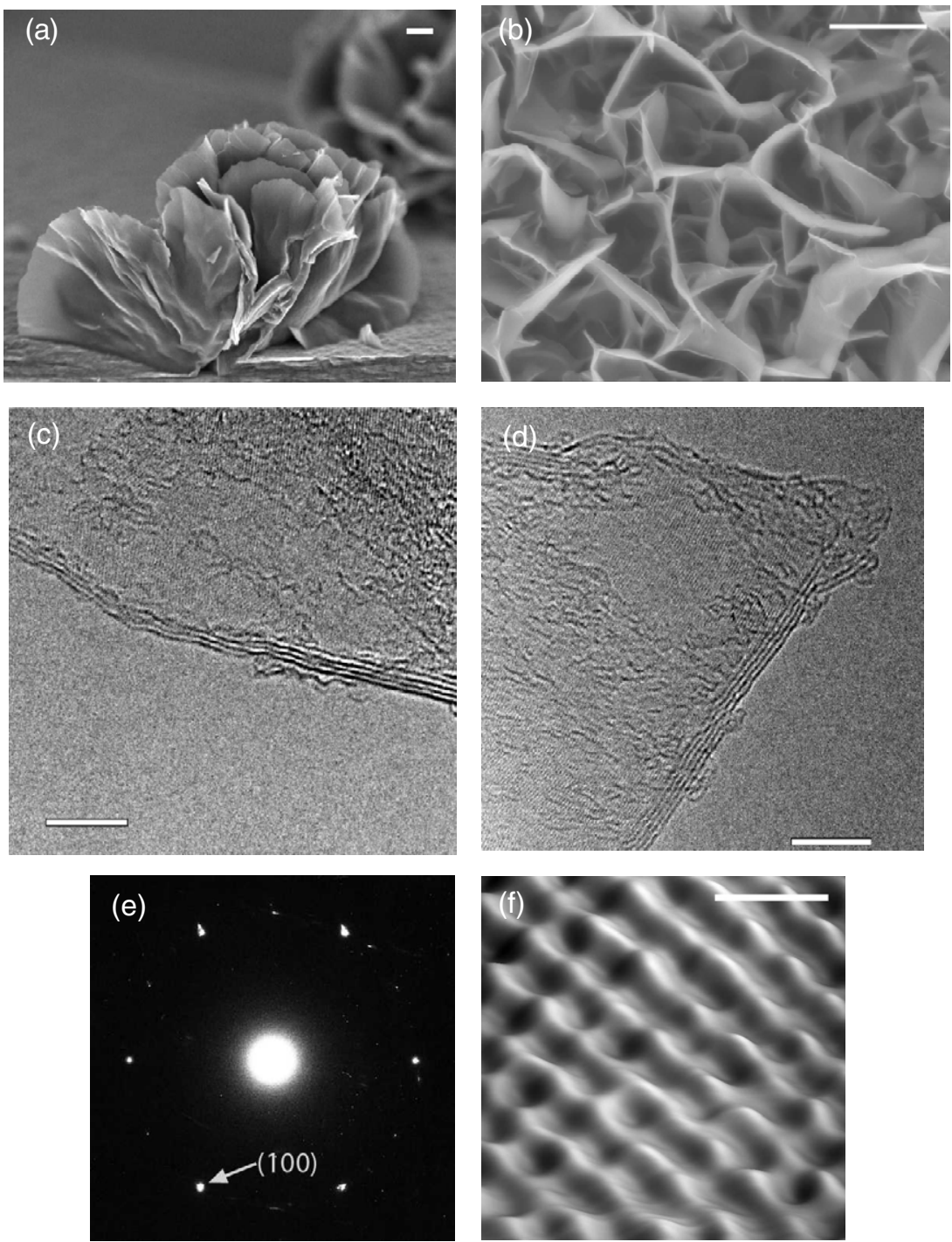

Figure 1. Structural characterization of aligned FLG. (a) Cross-sectional SEM image displays the typical dimensions and orientation of freestanding FLG. Note that the flakes are aligned vertically to the substrate surface. (b) Top view SEM image shows the high density of the flakes. Both SEM scale bars correspond to $1 \mu \mathrm{m}$. (c), (d) The average thickness of the flakes varies between 4 and 6 atomic layers, as revealed by TEM analysis. Both TEM scale bars correspond to $5 \mathrm{~nm}$. (e) A periodic hexagonal graphite crystal lattice structure is obtained by electron diffraction from an individual flake. The $\mathrm{C}(100)$ orientation is shown here. (f) The graphitic nature of the flakes is visualized with atomic resolution in low-temperature STM measurements. The scale bar in this topographic image corresponds to $0.5 \mathrm{~nm}$.

from edge effects [19] due to the orientation of FLG: vertically to the substrate surface and parallel to the incident laser beam. Graphene and FLG have a characteristic Raman signature in the 2D-band. The shape of the 2D-band, more specifically the absence of a typical graphite shoulder at $2680 \mathrm{~cm}^{-1}$, is a characteristic feature of FLG [20] and confirms the TEM analysis of the FLG thickness.

Straightforward and highly reproducible are the main advantages of our synthesis technique since it does not rely on the need for a metallic catalyst to drive the growth process, which implies that any material that withstands elevated temperatures can be used as a substrate. An essential requirement for the MW PECVD growth of FLG is a minimum temperature of $700^{\circ} \mathrm{C}$, since no flakes could be synthesized at temperatures below this threshold value. The substrates were heated purely by submersion in the hydrogen microwave plasma, which excludes the need for an expensive heating element but constrains the number of variable process parameters. The combination of microwave power, gas pressure and substrate position relative to the centre of the plasma need to be fixed in order to control the temperature. The remaining variable process parameters in our set-up are the substrate type, gas mixture and growth time. A broad range of materials was successfully used as substrates for the growth of FLG, including quartz, silicon and various other metals. The synthesis results on various substrates differ in adhesion of the flakes due to the possible formation of carbides on certain substrate surfaces as discussed below in more detail. A constant gas flow of methane diluted in hydrogen was typically used as the carbon source for the synthesis, but acetylene was found to yield similar results. It was observed that the growth process slows down and the average dimensions of the flakes 

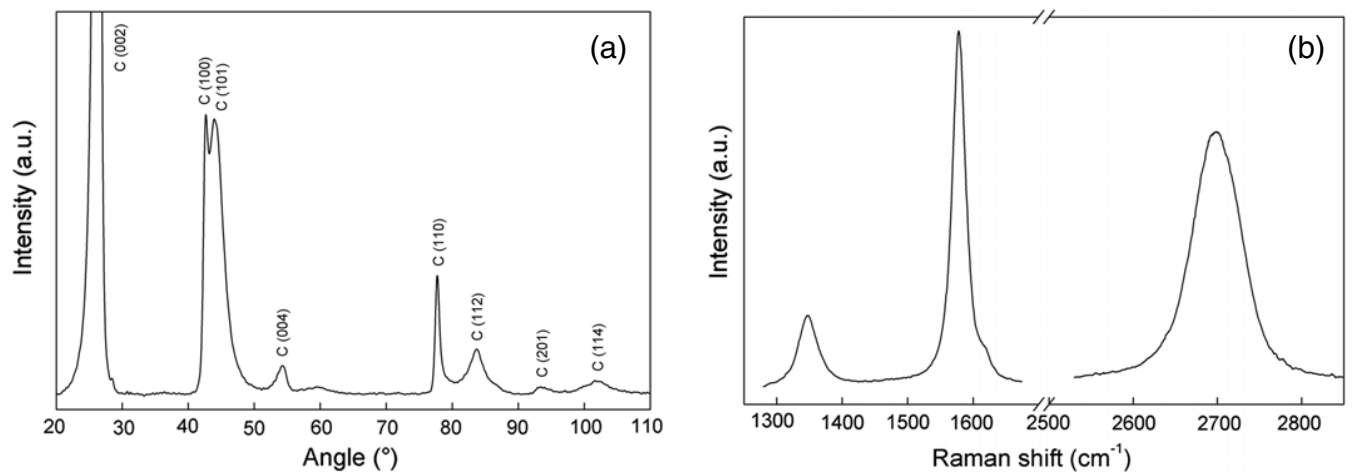

Figure 2. Qualitative analysis of as-grown FLG. (a) Several distinct graphite peaks can be identified in the XRD spectrum of FLG. The values for the inter-plane and the in-plane interatomic distance, extracted from the $\mathrm{C}(002)$ and $\mathrm{C}(110)$ peak positions, correspond to the tabulated values for graphite. (b) Raman spectroscopy identifies a weak $\mathrm{D}^{\prime}$-band around $1350 \mathrm{~cm}^{-1}$, a strong G-band around $1580 \mathrm{~cm}^{-1}$ and a strong 2D-band around $2700 \mathrm{~cm}^{-1}$. The intensity ratio of the D-band to the G-band varies between 0.20 and 0.30 for our FLG. The shape of the 2D-band is a characteristic feature of FLG.
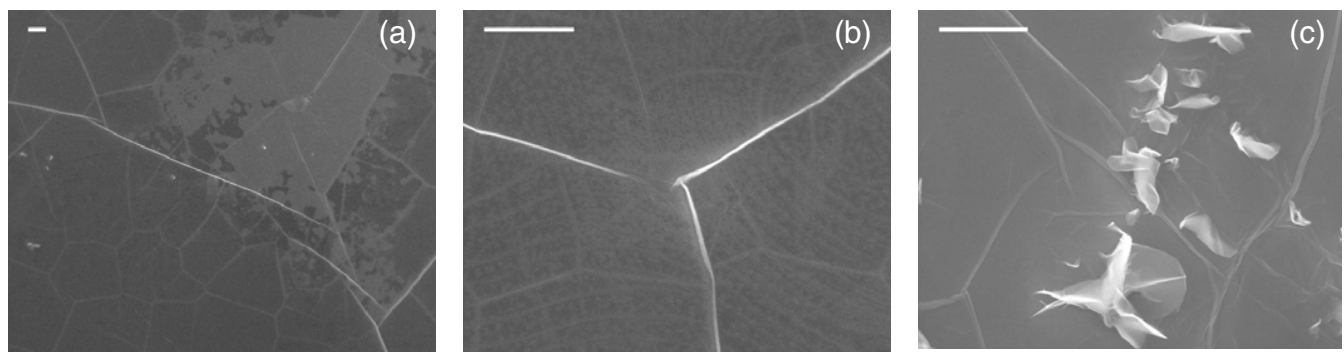

Figure 3. SEM analysis of the three-step growth mechanism of FLG. (a) In a first step, a graphite base layer is formed parallel to the substrate surface. Note that this layer shows many irregular cracks over its entire surface area. (b) The upward curling crack edges are the nucleation sites for the FLG in the second step. The growth direction is radically changed from parallel to vertical to the substrate surface. (c) The third step comprises the accumulation and incorporation of carbon radicals at the edges and sides of the freestanding FLG. The scale bars correspond to $1 \mu \mathrm{m}$.

decrease as the hydrogen gas flow increases relative to the methane flow, while keeping all other parameters constant. Therefore, in order to achieve the optimum result of several micrometres wide single or FLG, methane must be heavily diluted in hydrogen, while increasing the growth time to compensate for the otherwise small flake area. This way, a compromise between the average number of atomic layers and the size of the flakes can be found by adjusting the growth time and methane to hydrogen gas ratio.

\section{Discussion}

Scanning electron microscopy analysis of results with various synthesis times reveals a three-step growth mechanism for FLG. This is presented in figure 3, where the results are shown for platinum substrates. All other tested substrate materials yield similar results. In a first step, a base graphite layer is formed parallel to the substrate surface. This layer is not continuous but shows many irregular cracks over its entire surface area. Internal stress due to temperature gradients, ion bombardment and a mismatch between the lattice parameters of the substrate material and graphite are thought to be the main reasons for the formation of cracks [21, 22]. The density of the cracks increases with increasing growth time. Freestanding
FLG nucleates from these upward curling crack edges in the second step, radically changing their growth direction from parallel to vertical to the substrate surface. It takes less than $30 \mathrm{~s}$ after the initiation of the growth process by the introduction of methane in the plasma reactor to complete the first two steps. The third and final step comprises the accumulation and incorporation of carbon radicals at the edges of the freestanding FLG. This final step continues for several minutes, maintaining a constant gas flow in order to obtain micrometre-wide flakes.

The proposed stepwise growth mechanism is a general scheme, which can be further classified into different routes, depending on two properties of the substrate material, as investigated with XPS (see figure 4). First, if any stable carbides of the substrate material exist, they will be formed in an intermediate step before the nucleation of the base layer. This is an obvious conclusion with important consequences and is observed in the case of a silicon substrate, which shows a very strong carbide contribution at very short growth times, in contrast to the case of a quartz substrate. The carbide content in the XPS spectrum decreases with increasing growth time, since the base layer and FLG gradually cover the substrate. The formation of a carbide layer has the advantage of strongly increasing the adhesion of the flakes to the substrate surface, as tested with conventional cross-hatch 


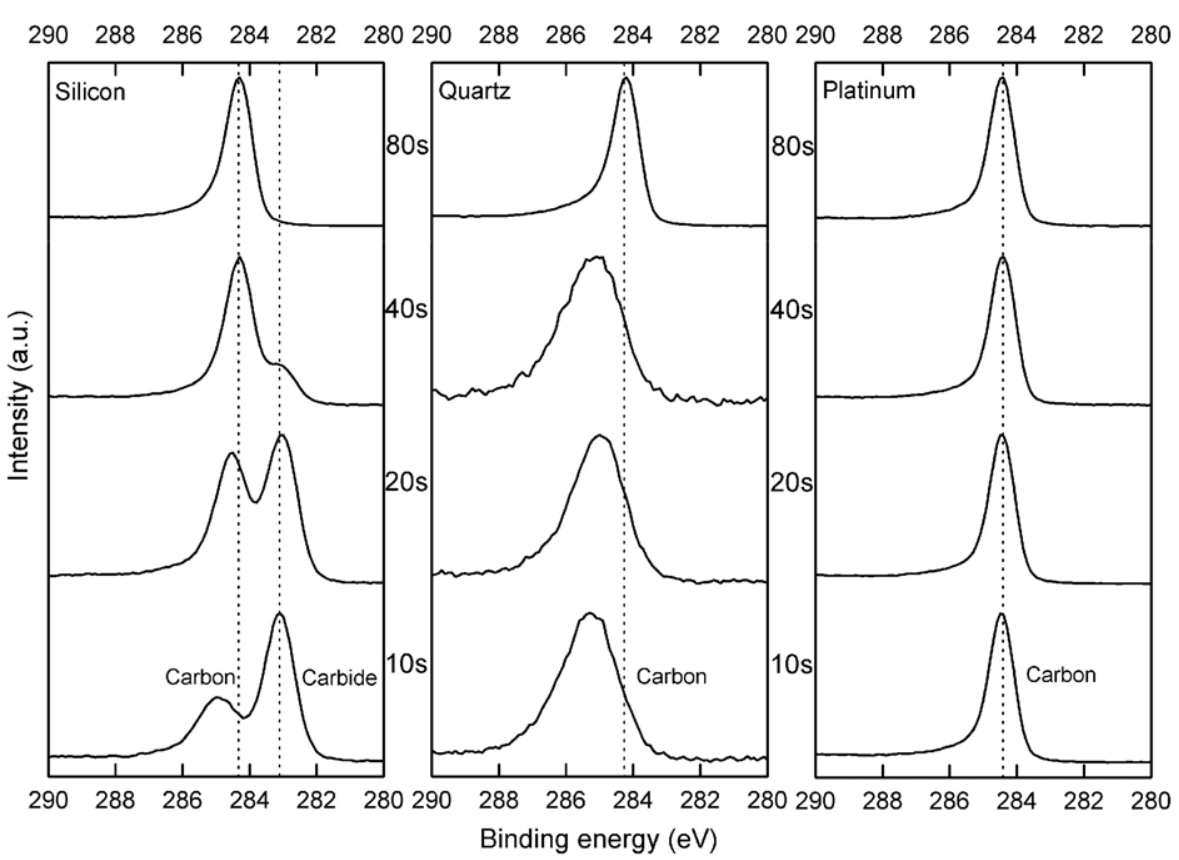

Figure 4. XPS analysis of the substrate-dependent evolution of the carbon coating in time. In the case of a silicon substrate, carbides are formed at the interface. In the case of silicon and quartz, a large mismatch between the lattice parameters of the substrate material and graphite causes an amorphous carbon interlayer to form between the substrate surface and the graphite base layer. This becomes visible as a clear shift of the carbon peak position towards higher binding energy at short growth times, accompanied by a Gaussian instead of asymmetric carbon peak shape. Note that there is no sign of amorphous carbon on platinum due to the good crystal lattice matching. As the growth time increases, the base layer and FLG cover the substrate surface and the carbide and amorphous carbon contribution to the spectra vanish. Eventually, all substrates show identical spectra.

adhesion test procedures by comparing results from silicon and quartz substrates. Second, if there exists a large mismatch between the lattice parameters of the substrate material and graphite, an amorphous carbon interlayer is formed as a buffer between the substrate surface and the graphite base layer. This is evident from the silicon and quartz spectra as a clear shift of the carbon peak position towards higher binding energy for short growth times, accompanied by a Gaussian instead of an asymmetric carbon peak shape. Amorphous carbon contains a combination of $\mathrm{sp}^{2}$ and $\mathrm{sp}^{3}$ bonds, which account for a higher binding energy and Gaussian peak shape [23]. There is no sign of a significant amount of amorphous carbon on platinum due to the good crystal lattice matching [24]. As the growth time increases, the base layer and FLG cover the substrate surface and the spectra of silicon, quartz and platinum no longer show any substrate-dependent features and all become identical. Even though the substrate material determines the chemical composition of the base layer and adhesion of the flakes, it does not interfere with the proposed three-step growth mechanism, which was found to be independent of the substrate material.

\section{Conclusions}

In conclusion, we present a possible route for the mass production of freestanding FLG by means of MW PECVD. Micrometre-tall flakes of 4 to 6 layers stacked graphene sheets were synthesized by controlled recombination of carbon radicals in a microwave plasma. This technique seems very promising since it is consistent with current industrial techniques, does not rely on the use of a catalyst and only requires substrate materials to withstand temperatures up to $700{ }^{\circ} \mathrm{C}$. An extensive characterization of FLG by scanning and transmission electron microscopy, x-ray diffraction, electron diffraction, STM and Raman spectroscopy is presented. From these results, it is concluded that the FLG are highly crystalline with few defects. Three steps in the FLG growth mechanism were identified, an important role of the substrate in the growth process was revealed and the adhesion of the flakes was found to be dependent on the possible formation of substrate carbides.

\section{Acknowledgments}

AM thanks M P Chowdhury for helpful discussions, $\mathrm{R}$ Kemps for SEM analysis and $\mathrm{M}$ Mertens for XRD analysis. The authors gratefully acknowledge funding from the Flemish Institute for Technological Research, the Belgian Interuniversity Attraction Poles (IAP) research programme and from the Fund for Scientific Research (FWO)-Flanders.

\section{References}

[1] Novoselov K S, Geim A K, Morozov S V, Jiang D, Zhang Y, Dubonos S V, Grigorieva I V and Firsov A A 2004 Electric field effect in atomically thin carbon films Science 306 666-9

[2] Novoselov K S, Jiang D, Schedin F, Booth T J, Khotkevich V V, Morozov S V and Geim A K 2005 Two-dimensional atomic crystals Proc. Natl Acad. Sci. USA 102 10451-3 
[3] Novoselov K S, Jiang Z, Zhang Y, Morozov S V, Stormer H L, Zeitler U, Maan J C, Boebinger G S, Kim P and Geim A K 2007 Room-temperature quantum Hall effect in graphene Science 3151137201

[4] Zhang Y, Tan Y, Stormer H L and Kim P 2005 Experimental observation of the quantum Hall effect and Berry's phase in graphene Nature $\mathbf{4 3 8}$ 201-4

[5] Katsnelson M I, Novoselov K and Geim A K 2006 Chiral tunnelling and the Klein paradox in graphene Nat. Phys. $2620-5$

[6] Novoselov K S, Geim A K, Morozov S V, Jiang D, Katsnelson M I, Grigorieva I V, Dubonos S V and Firsov A A 2005 Two-dimensional gas of massless Dirac fermions in graphene Nature 438 197-200

[7] Areshkin D A, Gunlycke D and White C T 2007 Ballistic transport in graphene nanostrips in the presence of disorder: importance of edge effects Nano Lett. 7 204-10

[8] Hass J, Feng R, Li T, Li X, Zong Z, de Heer W A, First P N, Conrad E H, Jeffrey C A and Berger C 2006 Highly ordered graphene for two-dimensional electronics Appl. Phys. Lett. 89143106

[9] Watcharotone S et al 2007 Graphene-silica composite thin films as transparent conductors Nano Lett. 7 1888-92

[10] Schedin F, Geim A K, Morozov S V, Hill E W, Blake P, Katsnelson M I and Novoselov K S 2007 Detection of individual gas molecules adsorbed on graphene Nat. Mater. 6 652-5

[11] Berger C et al 2004 Ultrathin epitaxial graphite: 2D electron gas properties and a route toward graphene-based nanoelectronics J. Phys. Chem. B 108 19912-6

[12] Gilje S, Han S, Wang M, Wang K L and Kaner R B 2007 A chemical route to graphene for device applications Nano Lett. 7 3394-8

[13] Wang Y Y, Gupta S and Nemanich R J 2004 Role of thin Fe catalyst in the synthesis of double- and single-wall carbon nanotubes via microwave chemical vapor deposition Appl. Phys. Lett. 85 2601-3
[14] Wu Y, Qiao P, Chong T and Shen Z 2002 Carbon nanowalls grown by microwave plasma enhanced chemical vapor deposition Adv. Mater. 14 64-7

[15] Hirao T, Ito K, Furuta H, Yap Y K, Ikuno T, Honda S, Mori Y, Sasaki T and Oura K 2001 Formation of vertically aligned carbon nanotubes by dual-RF-plasma chemical vapor deposition Japan. J. Appl. Phys. 40 L631

[16] Dresselhaus M S, Dresselhaus G, Saito R and Jorio A 2005 Raman spectroscopy of carbon nanotubes Phys. Rep. 40947

[17] Dresselhaus M S, Dresselhaus G, Jorio A, Souza Filho A G and Saito R 2002 Raman spectroscopy on isolated single wall carbon nanotubes Carbon $\mathbf{4 0} 2043$

[18] Kastner J, Pichler T, Kuzmany H, Curran S, Blau W, Weldon D N, Delamesiere M, Draper S and Zandbergen H 1994 Resonance Raman and infrared spectroscopy of carbon nanotubes Chem. Phys. Lett. 22153

[19] Graf D, Molitor F, Ensslin K, Stampfer C, Jungen A, Hierold C and Wirtz L 2007 Spatially resolved Raman spectroscopy of single- and few-layer graphene Nano Lett. $7238-42$

[20] Ferrari A C et al 2006 Raman spectrum of graphene and graphene layers Phys. Rev. Lett. 97187401

[21] Zhu M, Wang J, Holloway B C, Outlaw R A, Zhao X, Hou K, Schutthanandan V and Manos D M 2007 A mechanism for carbon nanosheet formation Carbon 45 2229-34

[22] Obraztsov A N, Obraztsova E A, Tyurnina A V and Zolotukhin A A 2007 Carbon 45 2017-21

[23] Reinke P, Garnier M G and Oelhafen P 2004 In situ photoelectron spectroscopy analysis of tetrahedral amorphous carbon films J. Electron. Spectrosc. Relat. Phenom. 136 239-45

[24] Ueta K, Saida M, Nakai C, Yamada Y, Sasaki M and Yamamoto S 2004 Highly oriented monolayer graphite formation on $\mathrm{Pt}(111)$ by a supersonic methane beam Surf. Sci. 560 183-90 